\title{
Association of A-20C polymorphism in the angiotensinogen gene with essential hypertension: a meta-analysis
}

\author{
R. Zeng ${ }^{1 *}$, Q.P. Wang ${ }^{2 *}$ M.X. Fang ${ }^{1}$, J. Zhuang ${ }^{3}$ and R.X. Fan ${ }^{3}$ \\ ${ }^{1}$ Department of Cardiovascular Surgery Intensive Care Unit, \\ Guangdong Provincial Cardiovascular Institute, Guangdong General Hospital, \\ Guangzhou, China \\ ${ }^{2}$ Department of Anesthesiology, Tianjin First Center Hospital, Tianjin, China \\ ${ }^{3}$ Department of Cardiovascular Surgery, \\ Guangdong Provincial Cardiovascular Institute, Guangdong General Hospital, \\ Guangzhou, China
}

\author{
*These authors contributed equally to this study. \\ Corresponding author: R.X. Fan \\ E-mail: fanrxme@163.com
}

Genet. Mol. Res. 14 (4): 12984-12992 (2015)

Received April 15, 2015

Accepted July 29, 2015

Published October 21, 2015

DOI http://dx.doi.org/10.4238/2015.October.21.19

\begin{abstract}
The A-20C polymorphism in the angiotensinogen (AGT) gene has been associated with increased risk of essential hypertension in several studies; however, these studies gave inconsistent results. In this study, we performed a meta-analysis to assess the association between AGT A-20C polymorphism and essential hypertension. Published literature was retrieved from PubMed. Pooled odd's ratio (OR) with 95\% confidence interval $(\mathrm{Cl})$ was calculated using fixed- or random-effect models. A total of 10 case-control studies containing 3653 cases and 3457 controls were enrolled to this meta-analysis. In a combined analysis, the results showed a significant association between the AGT A-20C polymorphism and risk of essential hypertension (AA vs CC: OR $=0.62,95 \% \mathrm{Cl}=0.46-0.84$; recessive model: $\mathrm{OR}=0.66,95 \% \mathrm{Cl}=0.49-0.88)$. In the subgroup analysis
\end{abstract}


stratified by race, significant associations were found between the AGT A-20C polymorphism and essential hypertension risk in Asians (AA vs CC: $\mathrm{OR}=0.59,95 \% \mathrm{Cl}=0.43-0.80$; recessive model: $\mathrm{OR}=0.63,95 \% \mathrm{Cl}=$ $0.46-0.85)$. In conclusion, the results of this meta-analysis suggested that the AGT A-20C polymorphism was associated with risk of essential hypertension in Asians.

Key words: Angiotensinogen gene; Essential hypertension; Meta-analysis; Polymorphism; A-20C

\section{INTRODUCTION}

Hypertension is a worldwide public health problem. According to the World Health Organization, hypertension can be attributed to the loss of 7.6 million lives annually (13.5\% of all deaths globally) and the loss of 57 million disability-adjusted life years worldwide (Arima et al., 2011). Recently, studies have been conducted to elucidate the pathological and physiological mechanism of hypertension; however, its molecular and genetic mechanism remains to be elucidated (Zhu et al., 2014). Extensive epidemiological studies have identified some environmental factors that may potentially result in essential hypertension, such as physical inactivity, obesity, a high sodium and low potassium diet, and alcohol consumption (Binder et al., 2007). In addition, genetic factors have received much attention over the past few years. A previous study has shown that genetic factors contribute up to $30-50 \%$ of the pathogenesis of this disorder (Newhouse et al., 2005).

The renin-angiotensin system (RAS) plays an important role in the regulation of blood pressure and cardiac function (Hall et al., 1986). Angiotensinogen (AGT) is the component of the RAS that is converted to angiotensin I by renin, and subsequently into angiotensin II. The AGT gene is located at lq42-43, and consists of five exons (Benigni et al., 2010). Previous meta-analyses have indicated an association between the M235T and T174M polymorphism in the AGT gene and an increased risk of hypertension (Mondry et al., 2005; Liao et al., 2014). However, the pathogenesis of the RAS gene in the involvement of hypertension could not be fully explained by these polymorphisms. In recent years, the A-20C polymorphism (rs5050, located in the promoter region of the AGT gene) has been reported to influence AGT transcriptional activity and serum AGT levels (Jeunemaitre et al., 1992).

To date, a large number of studies have attempted to examine the relationship between the AGT A-20C polymorphism and risk of hypertension. The results, however, differed between each of the studies. The discrepancies in the findings may be attributed to differences in the statistical power, and genetic and environmental factors (Wang and Pan, 2014). Therefore, in order to overcome the limitations of individual studies, we performed a meta-analysis to assess the association between the AGT A-20C polymorphism and essential risk of hypertension.

\section{MATERIAL AND METHODS}

\section{Selection of studies}

We performed a comprehensive search of all literature published before 1 December 2014 in PubMed (http://www.ncbi.nlm.nih.gov/pubmed) using the following key words: angiotensinogen or AGT, A-20C, polymorphism (mutation, or variant), and hypertension (or blood pressure or 
essential hypertension or $\mathrm{EH})$. We set no language restrictions. In case the search retrieved sequential or multiple articles with the same data, the data in the largest or most recent study was used. Disagreements were resolved by discussion and consensus. When the required data was absent, the corresponding authors were contacted via e-mail.

\section{Study selection}

Studies selected from this initial search were screened for eligibility based on the following criteria: use of a case-control or cohort study design; studies investigating the association between the AGT gene A-20C polymorphism and essential hypertension risk; studies providing sufficient information for the estimation of Odd's ratio (OR) with a 95\% confidence interval $(95 \% \mathrm{Cl})$; studies providing available data to acquire the genotype frequency of A-20C polymorphism. Studies were excluded if they: were meta-analyses, reviews, editorials, or comments; showed no available genotype frequency; and were duplicated studies.

\section{Data extraction}

Two reviewers ( $R$ Zeng and QP Wang) extracted the data independently and reached a consensus on all subjects. The following information was extracted from the included studies: first author, the year of publication, area, number of cases and controls, genotype frequencies in cases and controls, and evidence of Hardy-Weinberg equilibrium (HWE) in the controls (Table 1). For conflicting evaluations, disagreements were resolved following a discussion.

\section{Quality assessment}

The included studies whose genotype distribution in the controls was consistent with HWE $(P>0.10)$ were defined as high-quality studies, and those inconsistent with HWE $(P \leq 0.10)$ were characterized as low-quality studies (Salanti et al., 2005). Furthermore, we conducted a subgroup analysis according to the quality (high quality or low quality) to confirm if a deviation from HWE influenced the association in our meta-analysis.

\section{Statistical analysis}

The Fisher exact test (http://ihg.gsf.de/cgi-bin/hw/hwa1.pl) was used to assess the HWE. The association between the AGT A-20C polymorphism and essential hypertension was compared using the OR corresponding to a $95 \% \mathrm{Cl}$. In each study, the numbers of three genotypes (CC, AC, $\mathrm{AA}$ ) in the case and the control groups were used as pooled data. The pooled ORs were used to develop a a homozygote comparison (AA vs CC), a heterozygote comparison (AA vs AC), a dominant model $(C C+A C$ vs $A A)$, and a recessive model ( $A A+A C$ vs $C C)$. Heterogeneity was tested using the Q-test. The fixed effects model was used to pool the data when the $P$ value of $Q$-test was $>0.10$; otherwise, the random effects model was selected. A forest plot was generated for each analysis to summarize the statistical results. Subgroup analysis stratified according to racial descent and sample size was used to explore and explain the diversity among the results of different studies. Sensitivity analysis was performed by omitting one study at a time, to determine the magnitude of influence on the overall summary estimate (Zintzaras et al., 2005). Publication 
bias was evaluated by generating a Begg's funnel plot. All statistical analyses were performed with STATA v.12 (StataCorp LP, College Station, TX).

\section{RESULTS}

\section{Characteristics of included studies}

In total, 288 articles were identified as being relevant to the adopted keywords. Ten publications were included in the final meta-analysis (Ishigami et al., 1997; Wang et al., 1999; Sato et al., 2000; Tsai et al., 2003; Velez et al., 2006; Niu et al., 2007; Qi et al., 2008; Jiang et al., 2009; Ying et al., 2010; Li et al., 2013) based on the above-mentioned inclusion and exclusion criteria. Details of the selection process are presented in the flow chart in Figure 1. The baseline characteristics of all included studies are summarized in Table 1. These 10 case-control studies included a total of 3653 cases and 3457 healthy controls. All studies were case-control studies, which evaluated the association between the AGT A-20C polymorphism and essential hypertension risk. The year of publication of the included studies ranged from 1997 to 2014. All studies utilized blood samples for genotyping. The HWE test was performed on genotype distribution of the controls; all were high-quality studies, excluding those conducted by Sato et al. (2000), Tsai et al. (2003), and Niu et al. (2007).

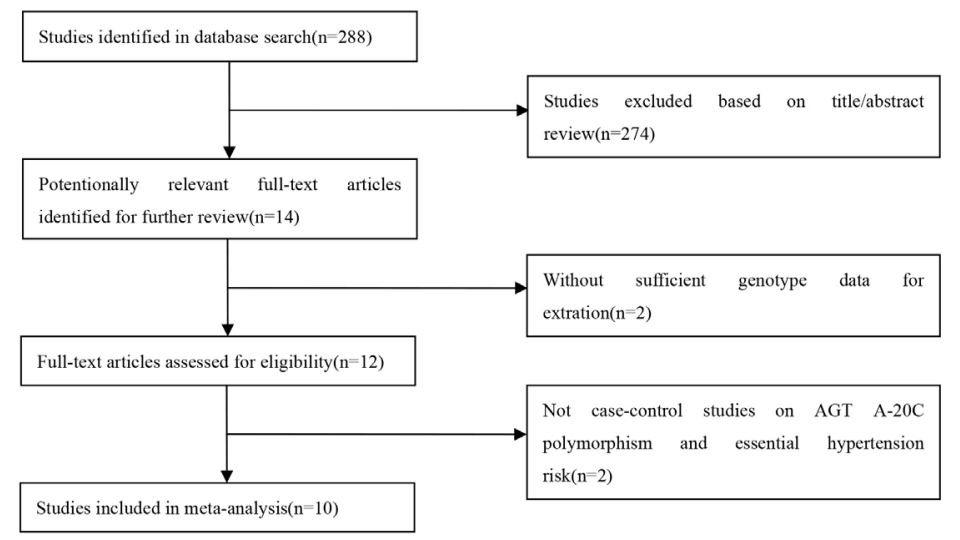

Figure 1. Flow diagram identifying the different studies included in this meta-analysis.

\begin{tabular}{|c|c|c|c|c|c|c|c|c|c|c|c|}
\hline \multirow[t]{2}{*}{ Study included } & \multirow[t]{2}{*}{ Area } & \multirow[t]{2}{*}{ Race } & \multirow[t]{2}{*}{ Cases/Controls } & \multicolumn{3}{|c|}{ Genotypes for cases } & \multicolumn{3}{|c|}{ Genotypes for controls } & \multirow[t]{2}{*}{ HWE test } & \multirow[t]{2}{*}{ Quality } \\
\hline & & & & $\overline{A A}$ & $A C$ & $\overline{\mathrm{CC}}$ & AA & $A C$ & $\mathrm{CC}$ & & \\
\hline Ishigami et al. (1997) & Japan & Asians & $76 / 158$ & 43 & 31 & 2 & 108 & 42 & 8 & 0.53 & High \\
\hline Wang et al. (1999) & Australia & Caucasians & $111 / 190$ & 84 & 25 & 2 & 120 & 65 & 5 & 0.09 & High \\
\hline Sato et al. (2000) & Japan & Asians & $180 / 195$ & 112 & 50 & 18 & 131 & 51 & 13 & 0.10 & Low \\
\hline Tsai et al. (2003) & China & Asians & $408 / 286$ & 379 & 23 & 6 & 284 & 1 & 1 & 0.48 & Low \\
\hline Velez et al. (2006) & America & Mixed & $308 / 155$ & 233 & 71 & 4 & 100 & 53 & 2 & 0.12 & High \\
\hline Niu et al. (2007) & China & Asians & $333 / 235$ & 216 & 113 & 4 & 132 & 97 & 6 & 0.54 & Low \\
\hline Qi et al. (2008) & China & Asians & $1122 / 965$ & 789 & 294 & 39 & 723 & 220 & 22 & 0.06 & High \\
\hline Jiang et al. (2009) & China & Asians & $220 / 235$ & 160 & 54 & 6 & 168 & 63 & 4 & 0.49 & High \\
\hline Ying et al. (2010) & China & Asians & $243 / 258$ & 166 & 64 & 13 & 174 & 76 & 8 & 0.37 & High \\
\hline Li et al. (2013) & China & Asians & $652 / 780$ & 192 & 144 & 16 & 600 & 168 & 12 & 0.54 & High \\
\hline
\end{tabular}

HWE = Hardy-Weinberg equilibrium . 


\section{Quantitative synthesis}

The combined results of the association between the A-20C polymorphism and essential hypertension risk are summarized in Figure 2 and Table 2. In this study, we found a statistically significant relationship between the A-20C polymorphism in the AGT gene and essential hypertension risk (AA vs CC: $\mathrm{OR}=0.62,95 \% \mathrm{Cl}=0.46-0.84$; the recessive model: $\mathrm{OR}=0.66$, $95 \% \mathrm{Cl}=0.49-0.88)$. In the subgroup analysis stratified by ethnicity (Table 2 ), we detected significant associations between the A-20C polymorphism and essential hypertension risk in Asians (AA vs $C C:$ OR $=0.59,95 \% \mathrm{Cl}=0.43-0.80$; the recessive model: $\mathrm{OR}=0.63,95 \% \mathrm{Cl}=0.46-0.85)$. Similar results were observed in the subgroup analysis based on a sample size $>500$ (recessive model: $\mathrm{OR}=0.58,95 \% \mathrm{Cl}=0.460-0.84$ ) and $\leq 500$ (recessive model: $\mathrm{OR}=0.66,95 \% \mathrm{Cl}=0.49-0.88$ ), and high-quality studies (AA vs CC: $\mathrm{OR}=0.59,95 \% \mathrm{Cl}=0.41-0.83$; recessive model: $\mathrm{OR}=0.63$, $95 \% \mathrm{Cl}=0.44-0.88)$. However, low quality studies showed a negative correlation between A-20C polymorphism and essential hypertension (Table 2).

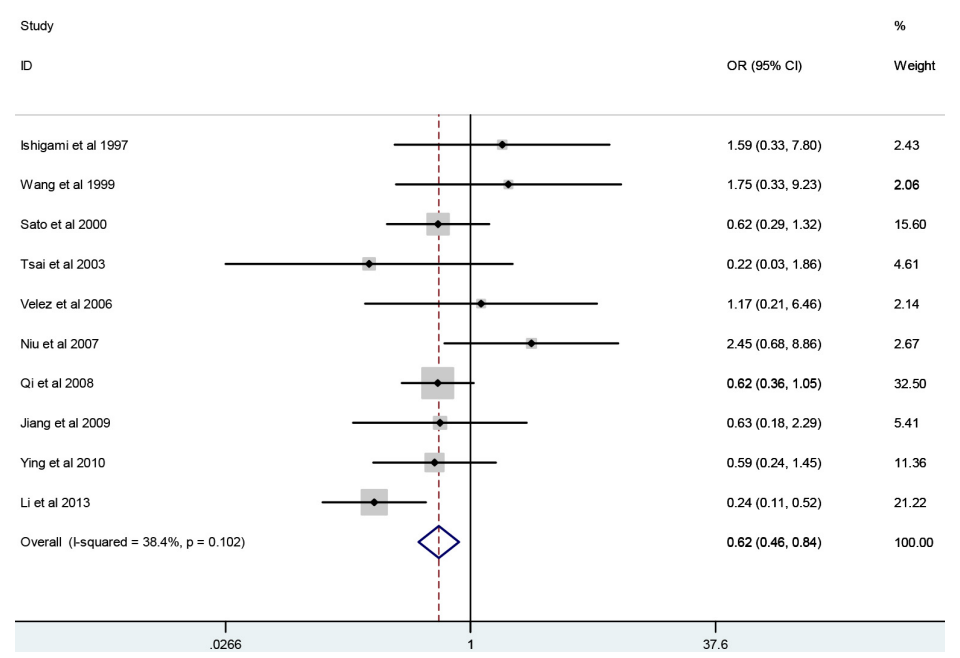

Figure 2. Meta-analysis of the association between the A20C polymorphism in the AGT gene and essential risk of hypertension (AA vs CC).

\begin{tabular}{|c|c|c|c|c|c|c|c|c|c|}
\hline \multirow[t]{2}{*}{ Variables } & \multirow[t]{2}{*}{$\mathrm{N}^{\mathrm{a}}$} & \multicolumn{2}{|c|}{ AA vs CC } & \multicolumn{2}{|l|}{$\mathrm{AA}$ vs AC } & \multicolumn{2}{|c|}{ Dominant model } & \multicolumn{2}{|c|}{ Recessive model } \\
\hline & & OR $(95 \% \mathrm{Cl})$ & $\mathrm{P}^{\mathrm{b}}$ & OR $(95 \% \mathrm{Cl})$ & $\mathrm{P}^{\mathrm{b}}$ & OR $(95 \% \mathrm{Cl})$ & $\mathrm{P}^{\mathrm{b}}$ & OR $(95 \% \mathrm{Cl})$ & $\mathrm{P}^{\mathrm{b}}$ \\
\hline Total & 10 & $0.62(0.46-0.84)$ & 0.10 & $0.90(0.63-1.29)$ & 0.00 & $1.16(0.81-1.67)$ & 0.00 & $0.66(0.49-0.88)$ & 0.31 \\
\hline \multicolumn{10}{|l|}{ Ethnicity } \\
\hline Asian & 8 & $0.59(0.43-0.80)$ & 0.09 & $0.76(0.52-1.11)$ & 0.00 & $1.38(0.94-2.01)$ & 0.00 & $0.63(0.46-0.85)$ & 0.23 \\
\hline Caucasian & 1 & $1.75(0.33-9.23)$ & - & $1.82(1.06-3.12)$ & - & $0.55(0.33-0.93)$ & - & $1.47(0.28-7.72)$ & - \\
\hline \multicolumn{10}{|l|}{ Sample size } \\
\hline$>500$ & 5 & $0.55(0.28-1.10)$ & 0.03 & $0.71(0.40-1.24)$ & 0.00 & $1.52(0.87-2.67)$ & 0.00 & $0.58(0.40-0.84)$ & 0.13 \\
\hline$\leq 500$ & 5 & $0.83(0.49-1.41)$ & 0.68 & $1.12(0.75-1.69)$ & 0.01 & $0.90(0.61-1.33)$ & 0.01 & $0.66(0.49-0.88)$ & 0.31 \\
\hline \multicolumn{10}{|l|}{ Quality } \\
\hline High-quality & 7 & $0.59(0.41-0.83)$ & 0.17 & $0.93(0.61-1.43)$ & 0.00 & $1.09(0.72-1.67)$ & 0.00 & $0.63(0.44-0.88)$ & 0.40 \\
\hline Low-quality & 3 & $0.75(0.41-1.37)$ & 0.09 & $0.75(0.32-1.78)$ & 0.00 & $1.54(0.62-3.82)$ & 0.00 & $0.75(0.42-1.36)$ & 0.14 \\
\hline
\end{tabular}




\section{Sensitivity analysis and publication bias}

One-way sensitivity analysis was performed to evaluate the stability of the meta-analysis (Tobias, 1999). The statistical significances of the overall results did not vary when any single study was omitted, indicating that the results of the meta-analysis were statistically robust (Figure 3 ). Begg's funnel plots were used to assess the publication bias of the included studies. The shape of funnel plots showed no evidence of publication bias (Figure 4).

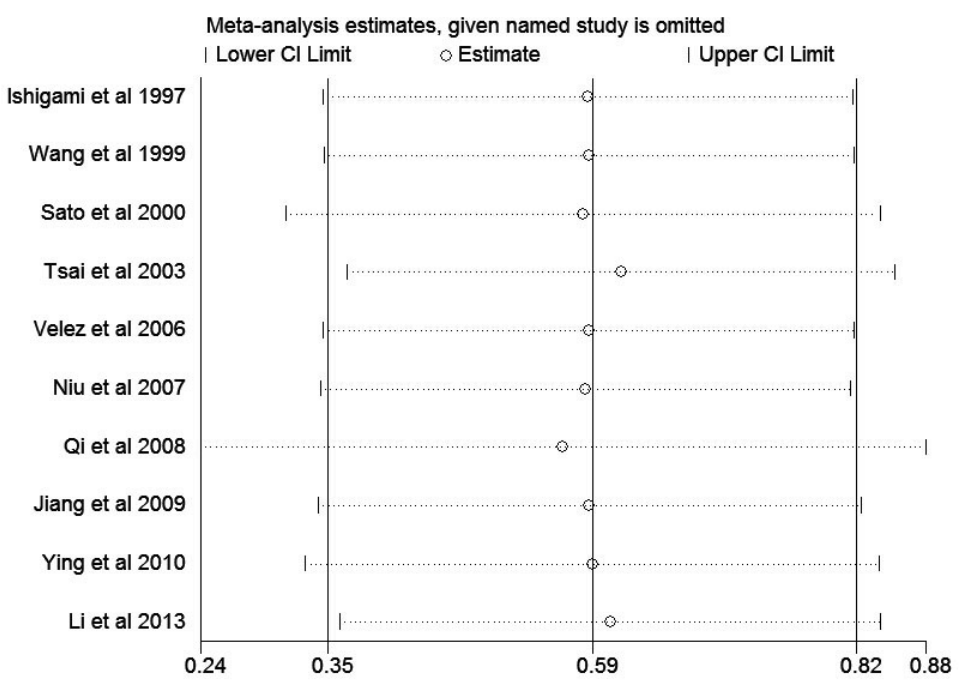

Figure 3. One-way sensitivity analysis of the pooled odds ratios and $95 \% \mathrm{Cls}$ for ACE I/D polymorphism, omitting each dataset in the meta-analysis (AA vs CC).

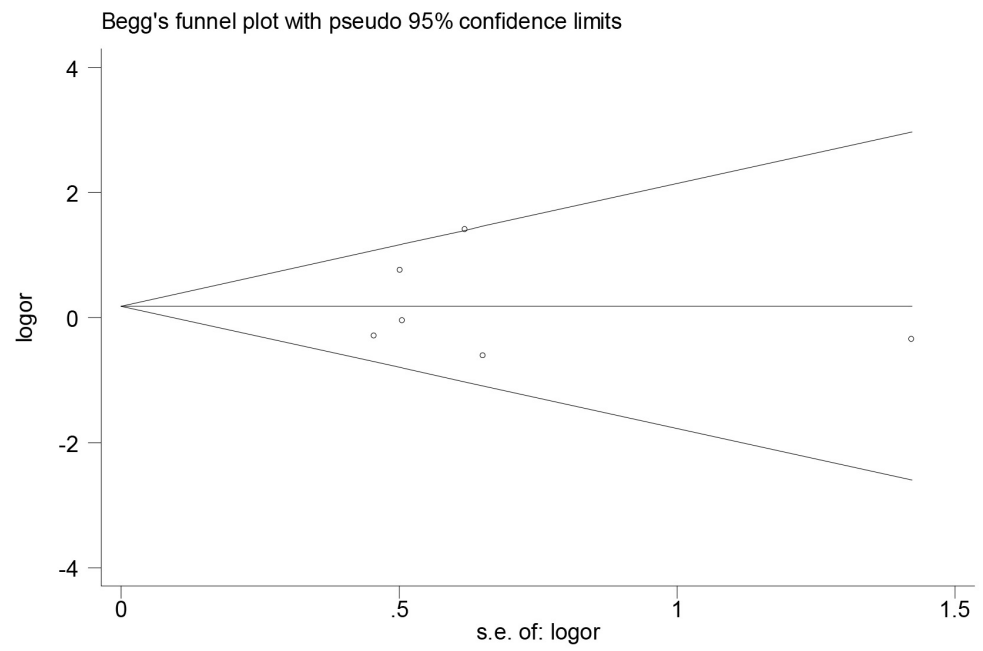

Figure 4. Publication bias for the association between the A20C polymorphism in the AGT gene and essential risk of hypertension (AA vs CC). 


\section{DISCUSSION}

Essential hypertension is a multifactorial disease that affects 20 to $30 \%$ of the population worldwide (Kearney et al., 2005). RAS plays a crucial role in the regulation of blood pressure. AGT is the major effector molecule of the RAS (Gu et al., 2011). Previous research has focused mainly on the M235T and T174M polymorphisms in the AGT gene (Mondry et al., 2005; Liao et al., 2014). Since M235T and T174M are located far from the rennin cleavage site, these 2 polymorphisms in the AGT gene do not fully explain the molecular mechanisms responsible for increased plasma AGT levels (Li et al., 2013). Ishigami et al. (1997) explored the association between the A-20C polymorphism and essential hypertension; the results of this analysis indicated that A-20C may be responsible for the increase in plasma AGT concentrations and in the development of essential hypertension (Ishigami et al., 1997). A number of recent studies have focused on the association between the A-20C polymorphism and essential hypertension. However, the results of these studies remain controversial. The most likely reason for these inconsistencies could be that they are single casecontrol studies conducted with small sample sizes. Therefore, we conducted a systematic review of the published findings and used meta-analysis techniques to quantitatively combine the results.

Our meta-analysis quantitatively assessed the association between the A-20C AGT polymorphism and essential hypertension risk in 3653 patients and 3457 controls. The results suggested the presence of a significant association between the AGT A-20C polymorphism and essential hypertension risk in the overall population. Because of the differences in genetic backgrounds and the environment in which the populations lived, we performed an ethnicity-specific subgroup analysis, and discovered a significant association between the A-20C polymorphism and essential hypertension risk in Asians. As only one study was performed in Caucasians, further investigation may be needed in Caucasians to verify these results. This meta-analysis also included several studies with a small sample size; this could have led to a selection bias. The results of this meta-analysis remained unaltered when stratified by sample size, indicating the lack of evidence of small-study bias in the meta-analysis. The quality of the included studies was assessed based on the $\mathrm{P}$ values of HWE in the control groups; the results of the meta-analysis were altered when lowquality studies were included. Therefore, low-quality studies may influence the association being investigated. Further, large and well-designed studies are required to confirm this conclusion.

The mechanism with which the A-20C polymorphism in the AGT gene relates to essential hypertension risk remains unclear. A previous study showed a significant correlation between the plasma AGT concentration and the A-20C polymorphism (Ishigami et al., 1997). AGT is a substrate of angiotensin II; we observed a highly significant positive correlation between the circulating AGT levels in the plasma and blood pressure (Walker et al., 1979). In addition, the potential function of the A-20C polymorphism might be affected via gene-gene interactions. The A-20C, M235T, A-6G, and G-217A polymorphisms displayed a strong linkage disequilibrium, which synergistically increased the risk of essential hypertension (Mondry et al., 2005). Further studies analyzing the gene-environment interactions should be taken into consideration in future analyses, which could lead to a comprehensive understanding of this disease.

Some limitations of our meta-analysis should be addressed. First, this meta-analysis was based on unadjusted OR estimates, as not all published studies presented adjusted ORs. In cases where the adjusted OR was presented, they were not adjusted by the same potential confounders, such as age, gender, and exposure. Secondly, the plasma AGT levels were not always available. Additionally, the effect of gene-gene and gene-environment interactions could not be addressed in this meta-analysis. 
In conclusion, this meta-analysis provides evidence that the A-20C polymorphism in the AGT gene may be associated with essential hypertension risk in Asians. Owing to the abovementioned limitations, the findings should be verified by further research in the near future.

\title{
Conflicts of interest
}

The authors declare no conflict of interest.

\section{ACKNOWLEDGMENTS}

\author{
Research supported by grants provided by the Guangdong Provincial Science and \\ Technology Project (\#2012A030400056) and Guangzhou Science and Technology Project \\ (\#2014Y2-00052).
}

\section{REFERENCES}

Arima H, Barzi F and Chalmers J (2011). Mortality patterns in hypertension. J. Hypertens. 29: S3-S7.

Benigni A, Cassis P and Remuzzi G (2010). Angiotensin II revisited: new roles in inflammation, immunology and aging. EMBO Mol. Med. 2: 247-257

Binder A (2007). A review of the genetics of essential hypertension. Curr. Opin. Cardiol. 22: 176-184.

Gu W, Liu Y and Wang Z (2011). Association between the angiotensinogen gene T174M polymorphism and hypertension risk in the Chinese population: a meta-analysis. Hypertens. Res. 35: 70-76.

Hall JE, Mizelle HL and Woods LL (1986). The renin-angiotensin system and long-term regulation of arterial pressure. $J$. Hypertens. 4: 387-397.

Ishigami T, Umemura S and Tamura K (1997). Essential hypertension and 5' upstream core promoter region of human angiotensinogen gene. Hypertension 30: 1325-1330.

Jeunemaitre X, Soubrier F, Kotelevtsev YV, Lifton RP, et al. (1992). Molecular basis of human hypertension: role of angiotensinogen. Cell 71: 169-180.

Jiang X, Sheng H, Li J, Xun P, et al. (2009). Association between renin-angiotensin system gene polymorphism and essential hypertension: a community-based study. J. Hum. Hypertens. 23: 176-181.

Kearney PM, Whelton M, Reynolds K, Muntner P, et al. (2005). Global burden of hypertension: analysis of worldwide data. Lancet 365: 217-223.

Li H, Du Z, Zhang L, Wu T, et al. (2013). The relationship between angiotensinogen gene polymorphisms and essential hypertension in a northern Han Chinese population. Angiology 65: 614-619.

Liao X, Yang Z, Peng D, Dai H, et al. (2014). Association of T174M polymorphism of angiotensinogen gene with essential hypertension: A meta-analysis. Genet. Mol. Biol. 37: 473-479.

Mondry A, Loh M, Liu P, Zhu AL, et al. (2005). Polymorphisms of the insertion/deletion ACE and M235T AGT genes and hypertension: surprising new findings and meta-analysis of data. BMC Nephrol. 6: 1.

Newhouse SJ, Wallace C, Dobson R, Mein C, et al. (2005). Haplotypes of the WNK1 gene associate with blood pressure variation in a severely hypertensive population from the British genetics of hypertension study. Hum. Mol. Genet. 14: 1805-1814.

Niu W, Qi Y, Cen W, Cui C, et al. (2007). Genetic polymorphisms of angiotensinogen and essential hypertension in a Tibetan population. Hypertens. Res. 30: 1129-1137.

Qi Y, Niu W, Zhou W, Hou S, et al. (2008). Correlation between angiotensinogen gene polymorphisms and essential hypertension in Chinese population. J. Hum. Hypertens. 22: 147-150.

Salanti G, Amountza G, Ntzani EE and loannidis JP (2005). Hardy-Weinberg equilibrium in genetic association studies: an empirical evaluation of reporting, deviations, and power. Eur. J. Hum. Genet. 13: 840-848.

Sato N, Katsuya T and Nakagawa T (2000). Nine polymorphisms of angiotensinogen gene in the susceptibility to essential hypertension. Life Sci. 68: 259-272.

Tobias A (1999). Assessing the influence of a single study in the meta-analysis estimate. Stata Tech. Bull. 8: 15-17.

Tsai CT, Fallin D, Chiang FT, Hwang JJ, et al. (2003). Angiotensinogen gene haplotype and hypertension: interaction with ACE gene I allele. Hypertension 41: 9-15. 
Velez DR, Guruju M, Vinukonda G, Prater A, et al. (2006). Angiotensinogen promoter sequence variants in essential hypertension. Am. J. Hypertens. 19: 1278-1285.

Walker WG, Whelton PK, Saito H, Russell RP, et al. (1979). Relation between blood pressure and renin, renin substrate, angiotensin II, aldosterone and urinary sodium and potassium in 574 ambulatory subjects. Hypertension 1: 287-291.

Wang WY, Glenn CL, Zhang W, Benjafield AV, et al. (1999). Exclusion of angiotensinogen gene in molecular basis of human hypertension: sibpair linkage and association analyses in Australian anglocaucasians. Am. J. Med. Genet. 87: 53-60.

Wang YJ and Pan Y (2014). The M235T polymorphism in the angiotensinogen gene and myocardial infarction risk: a metaanalysis. J. Renin Angiotensin Aldosterone Syst. 15: 294-300.

Ying CQ, Wang YH, Wu ZL, Fang MW, et al. (2010). Association of the renin gene polymorphism, three angiotensinogen gene polymorphisms and the haplotypes with essential hypertension in the Mongolian population. Clin. Exp. Hypertens. 32: 293-300.

Zhu Q, Guo Z, Hu X, Wu M, et al. (2014). Haplotype analysis of PPARy C681G and intron CT variants. Positive association with essential hypertension. Herz 39: 264-270.

Zintzaras E, Chatzoulis DZ, Karabatsas $\mathrm{CH}$ and Stefanidis I (2005). The relationship between C677T methylenetetrahydrofolate reductase gene polymorphism and retinopathy in type 2 diabetes: a meta-analysis. J. Hum. Genet. 50: 267-275. 